\title{
A case of Carney triad complicated by renal cell carcinoma and a germline SDHA pathogenic variant
}

\author{
Rachel Wurth ${ }^{D 1}$, Abhishek Jha2, Crystal Kamilaris(D1, Anthony J Gill33, Nicola Poplawski4,5, Paraskevi Xekouki1, \\ Martha M Quezado6, Karel Pacak², Constantine A Stratakis ${ }^{1}$ and Fady Hannah-Shmouni(D)
}

1Section on Endocrinology and Genetics, 2Section on Medical Neuroendocrinology, Eunice Kennedy Shriver National Institute of Child Health and Human Development, National Institutes of Health, Bethesda, Maryland, USA, ${ }^{3}$ Cancer Diagnosis and Pathology Group, Kolling Institute of Medical Research, Royal North Shore Hospital St Leonards NSW 2065 Australian and Sydney Medical School, University of Sydney, Sydney, New South Wales, Australia, ${ }^{4}$ Adult Genetics Unit, Royal Adelaide Hospital, ${ }^{5}$ Adelaide Medical School, University of Adelaide, Adelaide, South Australia, Australia, and 'Laboratory of Pathology Center for Cancer Research, National Cancer Institute, National Institutes of Health, Bethesda, Maryland, USA

Correspondence should be addressed to F Hannah-Shmouni Email

FADY.HANNAH-SHMOUNI@ NIH.GOV

\section{Summary}

Succinate dehydrogenase deficiency has been associated with several neoplasias, including renal cell carcinoma (RCC) and those associated with hereditary paraganglioma (PGL)/ pheochromocytoma (PHEO) syndromes, Carney dyad, and Carney triad. Carney triad is a rare multitumoral syndrome characterized by co-existing PGL, gastrointestinal stromal tumor (GIST), and pulmonary chondroma (CHO). We report a case of a 57-year-old male who presented with para-aortic and gastroesophogeal masses, and a right renal superior pole lesion, which were classified as multiple PGLs, a GIST, and a clear cell renal carcinoma, respectively, on pathology following surgical resection. Additionally, a CHO was diagnosed radiologically, although no biopsy was performed. A diagnosis of Carney triad was made. SDHB immunohistochemical staining was negative for the PGL and the GIST, indicating SDH-deficiency. Interestingly, the renal cell carcinoma (RCC) stained positive for both SDHB and SDHA. Subsequent genetic screening of SDH subunit genes revealed a germline inactivating heterozygous SDHA pathogenic variant (c.91 C>T, p.R31X). Loss of heterozygosity was not detected at the tumor level for the RCC, which likely indicated the SDHA variant would not be causative of the RCC, but could still predispose to the development of neoplasias. To the knowledge of the authors this is the first reported case of an SDHA pathogenic variant in a patient with Carney triad complicated by RCC.

\section{Learning points:}

- The succinate dehydrogenase enzyme is encoded by four subunit genes (SDHA, SDHB, SDHC, and SDHD; collectively referred to as SDHx), which have been implicated in several neoplasias and are classified as tumor suppressor genes.

- Carney triad is a rare multiple-neoplasia syndrome presenting as an association of PGLs, GISTs, and CHOs.

- Carney triad is most commonly associated with hypermethylation of SDHC as demonstrated in tumor tissue, but approximately $10 \%$ of cases are due to pathogenic $S D H x$ variants.

- Although SDHB pathogenic variants are most commonly reported in SDH-deficient renal cell carcinoma, SDHA disease-causing variants have been reported in rare cases. 


\section{Background}

Succinate dehydrogenase (SDH) is a heterotetrameric enzyme with dual roles in the mitochondrial citric acid cycle and electron transport chain. Germline inactivating $S D H B, S D H C$, and $S D H D$ pathogenic variants were implicated in the pathogenesis of paragangliomas (PGLs) and pheochromocytomas (PHEOs) in the early 2000s (1). In 2007, inherited gastrointestinal stromal tumors (GISTs) were additionally associated with $S D H B, S D H C$, and $S D H D$ pathogenic variants in patients with Carney dyad (CD), a rare autosomal-dominant disorder with co-existing PGL and GISTs) (1). It wasn't until 2010 that SDHA diseasecausing variants were implicated in hereditary PHEOs/ PGLs $(2,3)$.

Carney triad (OMIM\# 604287) was originally described by Dr J. Aidan Carney in 1977 and is a distinct entity from CD, presenting as an association of PGLs, GISTs, and pulmonary chondromas (CHOs, Fig. 1) (4). PHEOs have also been associated with the disease, but are not considered part of the 'triad'. Carney triad is sporadic with a female predilection and is typically diagnosed at a young age (median 18 years old) (5). The disease is predominantly associated with somatic DNA hypermethylation of SDHC as demonstrated in tumor tissue from these patients (6). However, in rare cases, $S D H A, S D H B, S D H C$, or $S D H D$ (collectively referred to as SDHx) germline pathogenic variants have been reported in patients with Carney triad $(2,3)$. It is hypothesized that

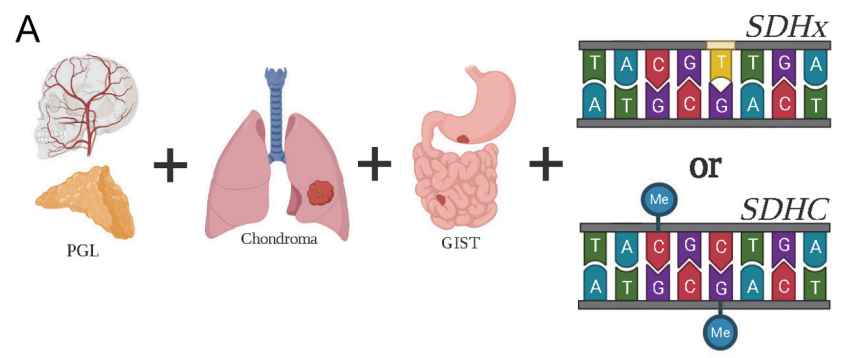

B

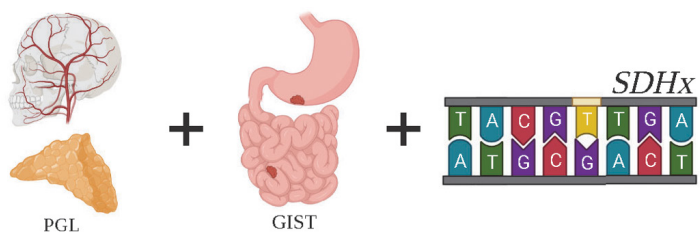

Figure 1

The presence of pulmonary chondromas discerns Carney triad and Carney dyad. (A) A diagnosis of Carney triad requires co-existing paraganglioma (PGL), pulmonary chondroma, and gastrointestinal stromal tumor (GIST) with either a germline pathogenic variant in SDHx or SDHC locus-specific hypermethylation. (B) In contrast, Carney dyad is the association of PGL and GIST with a germline pathogenic variant in SDHX. a predisposing germline disease-causing event in a non$S D H x$ gene is required for $S D H x$ pathogenic variants to cause Carney triad (3).

Pathogenic variants in SDHx have since been associated with several neoplasia syndromes (7). The three-P association (3PAs), which includes co-existing pituitary adenomas, PGLs, and PHEOs, has been associated with an SDHx disease-causing variant in approximately $43 \%$ of cases; the incidence increases to $75 \%$ in cases where the patient has a family history of PHEOs/PGLs (8). Pathogenic variants in SDHD and SDHC have been implicated in Cowden syndrome, a multipleneoplasia disorder characterized by benign and malignant breast, thyroid, and endometrial tumors $(7,9)$. In 1979, comorbid neuroblastoma, PHEO, and renal cell carcinoma (RCC) were reported in a single patient and later genetic analysis of affected family members implicated an $S D H B$ pathogenic variant (10). It has since been estimated that $1-5 \%$ of patients with RCC harbor a disease-causing variant in $S D H B$ or $S D H D(9)$.

In a handful of cases, germline SDHA pathogenic variants have been reported in patients with RCC, including a case with a co-existing GIST (11). In the current case, we report a patient with a germline $S D H A$ pathogenic variant in the setting of Carney triad complicated by RCC. The patient is one of the ten patients previously reported (patient number 4) in a review on SDHA-related metastatic PHEOs/PGLs (12).

\section{Case presentation}

A 57-year-old male diagnosed with hypertension at age 49 , presented with a gradual increase in blood pressure over a period of 12 months. Additional past history included coronary artery disease, hyperlipidemia, and tobacco use. Family history included hypertension, premature coronary artery disease, stroke, and prostate cancer, however, there was no family history of other tumors or endocrinopathies. He was initially started on Candesartan $4 \mathrm{mg}$ daily for treatment of hypertension, however, his hypertension persisted resulting in a gradual increase to $32 \mathrm{mg}$ daily and the addition of hydrochlorothiazide (12.5 $\mathrm{mg}$ daily), with the eventual switch to telmisartan $40 \mathrm{mg}$ daily and amlodipine 5 mg daily. For seven years following his diagnosis of hypertension, the patient experienced episodic increases in blood pressure, to a systolic pressure greater than 180 $\mathrm{mmHg}$ associated with a tight band sensation around his forehead lasting half a day. These episodes occurred every 1-2 months and required emergency care on two 
occasions. He was referred to an endocrinologist for further evaluation.

\section{Investigation}

Clinical and biochemical evaluation for endocrine causes of secondary hypertension was performed including for PHEO/PGL and primary aldosteronism, and revealed plasma metanephrines and 24-h urine catecholamines and metanephrines within the normal range. An elevated aldosterone-to-renin ratio (55) was noted and the patient underwent a saline suppression test which was not consistent with primary aldosteronism $(4 \mathrm{~h}$ serum aldosterone $<5 \mathrm{ng} / \mathrm{dL}$ ). Without apparent signs of endocrine-related hypertension, the episodic blood pressure exacerbations were believed to be anxiety related. He also underwent a $1 \mathrm{mg}$ overnight dexamethasone suppression test that was not consistent with hypercortisolemia (serum cortisol after dexamethasone $<1.8 \mu \mathrm{g} / \mathrm{dL})$.

Abdominal CT revealed a left adrenal adenoma, a 5.1 $\mathrm{cm}$ para-aortic mass (Fig. 2A), and a right renal superior pole lesion measuring $2.5 \mathrm{~cm}$, which was suspicious for a carcinoma (Fig. 2B). 123I-metaiodobenzylguanidine (123I-MIBG, arrow in Fig. 2C) and ${ }^{18} \mathrm{~F}-$ fluorodeoxyglucosePET ( ${ }^{18}$ F-FDG-PET, arrow in Fig. 2D) scans were performed, which suggested the para-aortic mass to be consistent with a PGL. Additionally, ${ }^{18} \mathrm{~F}-\mathrm{FDG}$ uptake was noted in the gastroesophageal region (dashed arrow in Fig. 2D). The left adrenal mass was not associated with ${ }^{123}$ I-MIBG (Fig. 2C) or ${ }^{18}$ F-FDG activity (Fig. 2D) and was not suspected to be hormonally active per prior biochemical testing.

\section{Treatment}

The patient underwent an uncomplicated surgical resection of the PGL and a partial right nephrectomy. The gastroesophogeal region where ${ }^{18} \mathrm{~F}-\mathrm{FDG}$ uptake (dashed arrow in Fig. 2D) had been noted was inspected and two subcentimeter nodules were found on the wall of the stomach. A subsequent gastroscopy was performed to resect the nodule nearest the gastroesophogeal junction.

\section{Outcome and follow-up}

The resected PGL (arrows in Fig. 2A, B, C and D) measured $4.2 \mathrm{~cm}$ in length and immunohistochemisty (IHC) staining was performed for SDHA and SDHB, which was positive and negative, respectively. Plasma 3-methoxytyramine levels were performed prior to surgery and were not elevated $(<200 \mathrm{pmol} / \mathrm{L})$. Pathology from the renal lesion was consistent with a $2.3 \mathrm{~cm}$ conventional clear cell renal carcinoma, and did not demonstrate eosinophilic cytoplasm or vacuolated cytoplasm which are typically associated with SDH-deficient RCC (13) (Fig. 3A, B, C and D). Of note, IHC staining was positive for both SDHB and SDHA for the RCC (Fig. 3C and D). SDHC hypermethylation was not detected in the PGL, GIST, or RCC. The ${ }^{18} \mathrm{~F}-\mathrm{FDG}-$ avid nodule (dashed arrow, Fig. 2D) resected from the gastroesophogeal junction was consistent with a GIST, which stained positive for SDHA and negative for SDHB on IHC. A 3-month post-operative endoscopic ultrasound was remarkable for two submucosal lesions $(1.2 \mathrm{~cm}$ and $5 \mathrm{~mm}$ ) at the gastroesophogeal junction. Fine needle biopsies were taken and pathology was inconclusive
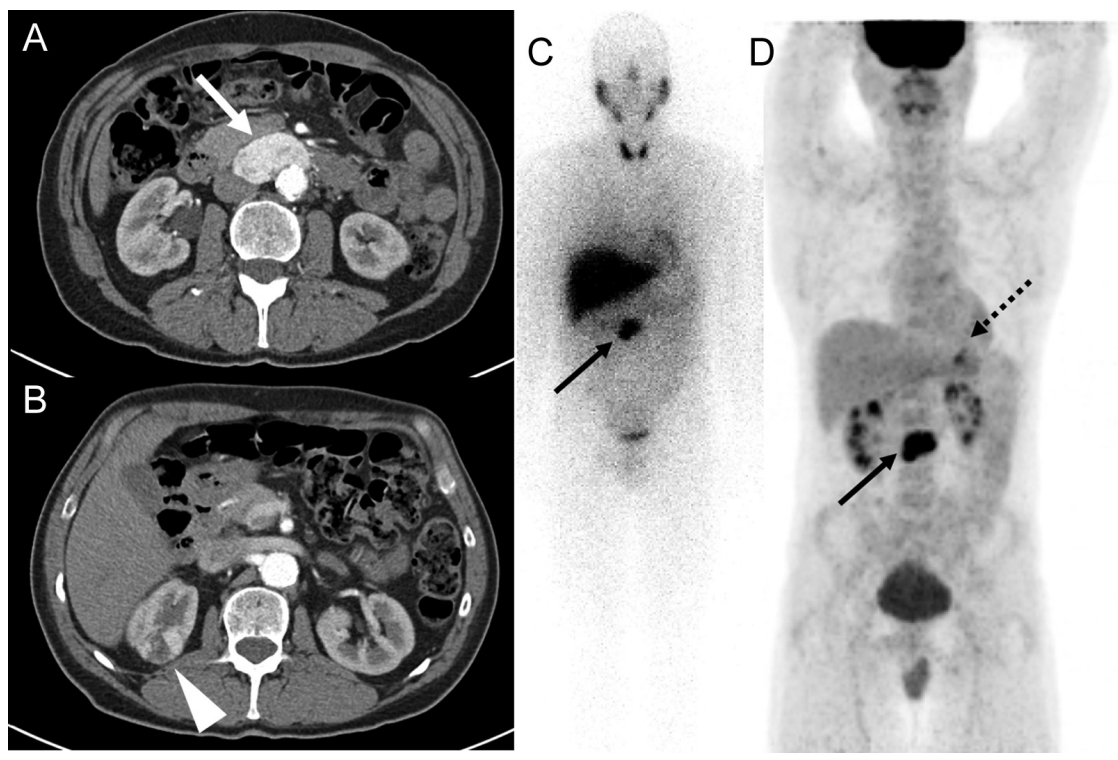

Figure 2

In this figure, 57-year-old-male, axial images of contrast-enhanced $\mathrm{CT}$ ( $\mathrm{A}$ and $\mathrm{B}$ ) demonstrates a para-aortic mass (arrow, A) and right superior pole lesion (arrowhead, B). The anterior planar image of 1231-metaiodobenzylguanidine demonstrates uptake in para-aortic mass (arrow, C) which is also seen on the anterior maximum intensity projection of 18F-fluorodeoxyglucose (18F-FDG) PET scan (arrow, D). The 18F-FDG PET scan also demonstrates uptake in gastroesophageal lesion (dashed arrow, D). 


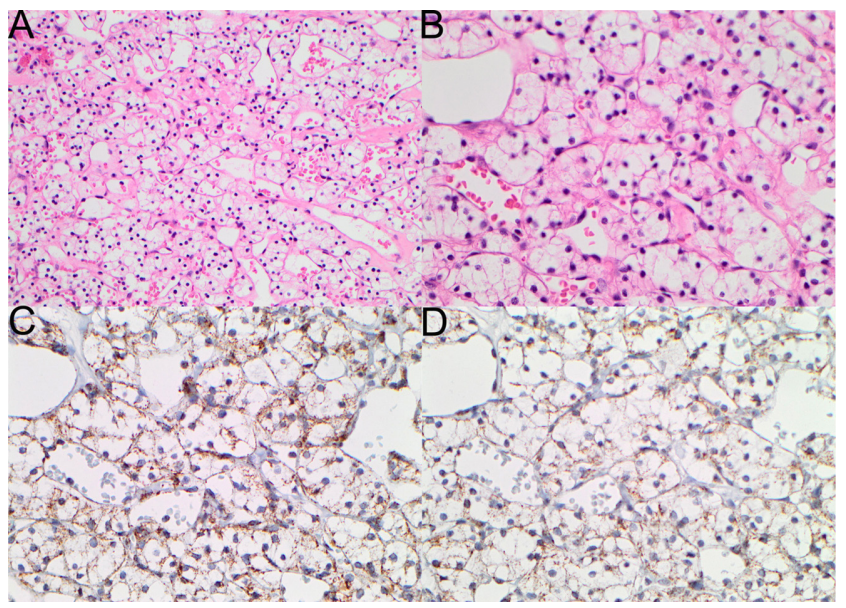

\section{Figure 3}

Hematoxylin and eosin stain sections show a classical renal cell carcinoma, clear cell type, low grade (ISUP nuclear/nucleolar grade 2, (A and B) $200 \times$ and 400× magnification, respectively). SDHA (C) and SDHB (D) immunohistochemical stains exhibit positive, granular, cytoplasmic staining (preserved expression, 400×).

for GISTs. Chest CT demonstrated a right middle lobe lung lesion suspicious for $\mathrm{CHO}$, although no biopsy was performed.

The aforementioned findings led to a diagnosis of Carney triad. Initial genetic screening for $S D H B, S D H C$, and SDHD pathogenic variants was unremarkable, however, follow-up sequencing of SDHA revealed a germline heterozygous pathogenic variant (c.91 C>T, p.R31X). Whole-exome sequencing did not reveal pathogenic variants in other genes associated with RCC and chromosome analysis by comparative genomic hybridization (CGH) was normal. This variant has previously been reported in a patients with hereditary PHEO/PGL and results in loss of function of SDHA (14). DNA sequencing of the RCC, GIST, and PGL did not indicate loss of heterozygosity $(\mathrm{LOH})$ at the variant region of interest. The patient's daughter, who is in her third decade of life, has inherited the pathogenic variant in
SDHA, however, she had no evidence of disease. She was counseled to undergo annual biochemical catecholamine and metanepherine screening and whole body scans for the appearance of Carney triad-related tumors.

The patient was advised to obtain bilateral renal ultrasound, esophagogastroduodenoscopies, alternating whole-body CT and MRI annually, and functional imaging every 2 years as surveillance for recurrence and appearance of tumors associated with Carney triad. Two years after surgical resection of the PGL, functional imaging revealed a mediastinal lesion on both ${ }^{18} \mathrm{~F}-\mathrm{FDG}$ PET/CT and ${ }^{68} \mathrm{Ga}$-DOTATATE-PET/CT scans (Fig. $4 \mathrm{~A}$ and B, dashed arrows), and a left carotid body tumor which was only detected on ${ }^{68} \mathrm{Ga}$-DOTATATE (Fig. 4B, arrow). Both lesions remained stable at 4 years after initial surgery (Fig. $4 \mathrm{C}$ and D, ${ }^{18}$ F-FDG and ${ }^{68} \mathrm{Ga}$-DOTATATE, respectively). His hypertension was well controlled with telmisartan $40 \mathrm{mg}$ and amlodipine $5 \mathrm{mg}$ combination daily.

\section{Discussion}

Carney triad is a rare multiple neoplasia disorder characterized by comorbid PGLs, GISTs, and CHOs. Although not considered part of the 'triad', PHEOs have also been associated with the disease (1). SDH enzymatic activity was found to be decreased in tumors from these patients, however, $S D H x$ pathogenic variants had not been reported (3). Further studies of SDH-deficient tumors from patients with Carney triad indicated SDHC locus-specific hypermethylation may be causative of diminished SDH enzymatic activity (15). Our group has reported differential DNA methylation of $S D H B$ and $S D H C$, without notable methylation of SDHA or SDHD. Interestingly, while the methylation pattern of $S D H C$ is sequence-specific for Carney triad and consistent between patients, methylation of $S D H B$ when seen in the triad has no apparent pattern, and is similar to that seen in other
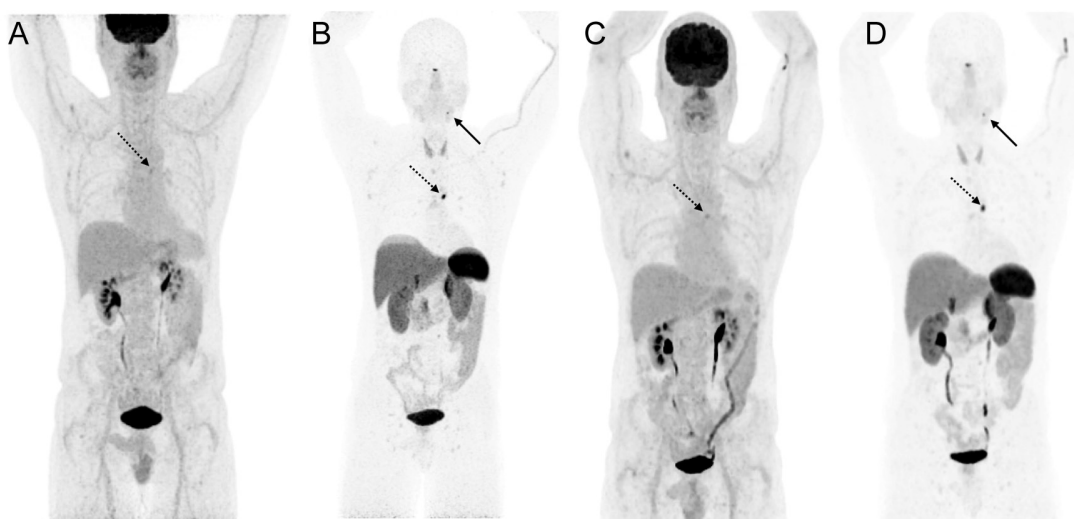
Figure 4
The follow-up functional imaging with 18F-FDG (A and $C$ ) and 68Ga-DOTATATE (B and D) PET imaging after $2(A$ and $B)$ and $4(C$ and $D)$ years of paraganglioma surgery demonstrates stable mediastinal lesion (dashed arrows, A, B, C and D) on both 18F-FDG and 68Ga-DOTATATE PET scans whereas left carotid body tumor only on 68Ga-DOTATATE (arrows, B and D). Both the lesions were not localized on anatomic imaging by MRI.


SDH-deficient tumors (6). Thus, SDHC-sequence-specific methylation is considered the molecular signature for Carney triad and is used diagnostically in our laboratory.

Although the majority of Carney triad patients have site-specific SDHC methylation, approximately $10 \%$ of cases harbor pathogenic germline variants in $S D H x$ (2). It is hypothesized a predisposing pathogenic germline event in a non-SDHx gene, in addition to the $S D H x$ diseasecausing variant, is required to cause the disease (3). Interestingly, half of the reported Carney triad patients with $S D H x$ pathogenic variants were males, in contrast to the associated female predilection. No other phenotypic differences were noted. Additionally, the variants were inherited, although with incomplete penetrance (2). In our case, we report a male patient with an inactivating SDHA pathogenic variant, which was inherited by his daughter, who has not developed any manifestations of Carney triad to date. Considering the low penetrance of SDHA disease-causing variants, estimated at less than 5\% in patients with PHEO/PGL's, it is unclear if the patient's daughter will develop tumors associated with Carney triad (16). Additionally, it is important to acknowledge we are unable to confirm the $S D H A$ variant is causative of the patient's phenotype. It is possible our testing failed to identify a variant in one of the other $S D H x$ genes. Given the normal SDHA IHC and low penetrance of SDHA pathogenic variants, we hypothesize this variant may be only one of the $S D H x$ drivers involved in the Carney triad phenotype.

The SDH enzyme is a membrane-associated mitochondrial protein complex that functions as a key component of the electron transport chain. Although impaired aerobic respiration has been consistently observed in neoplastic cells, it has largely been regarded as an association rather than a cause of disease. However, the implication of $S D H x$ pathogenic variants in hereditary PHEOs/PGLs altered this notion and has led to the classification of SDHx as tumor suppressor genes (17). Both the PGL and GIST in our patient were SDH-deficient, as indicated by negative SDHB IHC staining, which is consistent with previous reports of Carney triad patients harboring $S D H x$ pathogenic variants (2).

Both familial and sporadic RCC have been associated with $S D H x$ pathogenic variants, most commonly affecting $S D H B$ or $\operatorname{SDHD}(9,18)$. IHC staining for SDHB is often used to screen for the presence of SDH-deficient tumors; the presence of SDHx pathogenic variants or SDH epigenetic alterations will result in diminished SDHB protein levels. Interestingly, in the present case, SDHB and SDHA staining were positive in the RCC in contrast to the other associated tumors which were SDHB negative. The preserved expression of SDHA and SDHB in conjunction with histological characteristics consistent with clear cell renal carcinoma indicates this tumor may be an incidental finding. In the patient harboring a pathogenic variant in SDHA reported by Jiang et al., IHC staining of the renal tumor was positive for SDHA and negative for SDHB. Additionally, $\mathrm{LOH}$ was not detected in the RCC which is in agreement with our findings. Our report provides further support to the increasing evidence that $S D H x$ pathogenic variants may predispose patients to develop renal neoplasms (19), although the exact role of $S D H A$ disease-causing variants in the pathogenesis of RCC remains unknown.

In conclusion, we report a novel presentation of a germline inactivating $S D H A$ pathogenic variant in a patient with Carney triad complicated by RCC. Although we believe the SDHA disease-causing variant is one of the drivers of the patient's Carney triad phenotype, it is unclear if this variant is causative of the RCC. A small number of cases have reported SDHA disease-causing variants co-existing with RCC, including in a patient with comorbid GIST (11). However, to the knowledge of the authors, none of the reported cases involved a patient with Carney triad.

\section{Declaration of interest}

Constantine Stratakis holds patents on the PRKAR1A, PDE11A, and the GPR101 genes and his laboratory is the recipient of past and ongoing research grant support from Pfizer, Inc. Karel Pacak and Constantine A. Stratakis are Senior Editors of Endocrinology, Diabetes and Metabolism Case Reports. Karel Pacak and Constantine A Stratakis were not involved in the review or editorial process for this paper, on which they are listed as authors.

\section{Funding}

This work was supported by the research project Z01-HD008920 (Principal Investigator: Constantine A Stratakis) of the Intramural Research Program of the Eunice Kennedy Shriver National Institute of Child Health \& Human Development (NICHD), National Institutes of Health (NIH), Bethesda, MD, USA.

\section{Patient consent}

Written informed consent has been obtained from the patient for publication of the submitted article and accompanying images.

\section{Author contribution statement}

All co-authors contributed equally.

\section{Acknowledgement}

The authors like to thank Dr George Tallis who jointly cared for the patient. 


\section{References}

1 McWhinney SR, Pasini B, Stratakis CA \& International Carney Triad and Carney-Stratakis Syndrome Consortium. Familial gastrointestinal stromal tumors and germ-line mutations. New England Journal of Medicine 2007357 1054-1056. (https://doi.org/10.1056/ NEJMc071191)

2 Boikos SA, Xekouki P, Fumagalli E, Faucz FR, Raygada M, Szarek E, Ball E, Kim SY, Miettinen M, Helman LJ, et al. Carney triad can be (rarely) associated with germline succinate dehydrogenase defects. European Journal of Human Genetics 201624 569-573. (https://doi. org/10.1038/ejhg.2015.142)

3 Settas N, Faucz FR \& Stratakis CA. Succinate dehydrogenase (SDH) deficiency, Carney triad and the epigenome. Molecular and Cellular Endocrinology 2018469 107-111. (https://doi.org/10.1016/j. mce.2017.07.018)

4 Carney JA, Sheps SG, Go VL \& Gordon H. The triad of gastric leiomyosarcoma, functioning extra-adrenal paraganglioma and pulmonary chondroma. New England Journal of Medicine 1977296 1517-1518. (https://doi.org/10.1056/NEJM197706302962609)

5 Carney JA. Carney triad. Frontiers of Hormone Research 201341 92-110. (https://doi.org/10.1159/000345672)

6 Haller F, Moskalev EA, Faucz FR, Barthelmeß S, Wiemann S, Bieg M, Assie G, Bertherat J, Schaefer IM, Otto C, et al. Aberrant DNA hypermethylation of SDHC: a novel mechanism of tumor development in Carney triad. Endocrine-Related Cancer 201421 567-577. (https://doi.org/10.1530/ERC-14-0254)

7 Xekouki P, Brennand A, Whitelaw B, Pacak K \& The SCA. 3PAs: an update on the association of pheochromocytomas, paragangliomas, and pituitary tumors. Hormone and Metabolic Research 201951 419-436. (doi: 10.1055/a-0661-0341)

8 Xekouki P, Szarek E, Bullova P, Giubellino A, Quezado M, Mastroyannis SA, Mastorakos P, Wassif CA, Raygada M, Rentia N, et al. Pituitary adenoma with paraganglioma/pheochromocytoma (3PAs) and succinate dehydrogenase defects in humans and mice. Journal of Clinical Endocrinology and Metabolism 2015100 E710-E719. (https://doi.org/10.1210/jc.2014-4297)

9 Ni Y, Zbuk KM, Sadler T, Patocs A, Lobo G, Edelman E, Platzer P, Orloff MS, Waite KA \& Eng C. Germline mutations and variants in the succinate dehydrogenase genes in Cowden and Cowden-like syndromes. American Journal of Human Genetics $2008 \mathbf{8 3} 261-268$. (https://doi.org/10.1016/j.ajhg.2008.07.011)

10 Fairchild RS, Kyner JL, Hermreck A \& Schimke RN. Neuroblastoma, pheochromocytoma, and renal cell carcinoma. Occurrence in a single patient. Journal of the American Medical Association 1979242 2210-2211. (https://doi.org/10.1001/jama.1979.03300200040021)

11 Jiang Q, Zhang Y, Zhou YH, Hou YY, Wang JY, Li JL, Li M, Tong HX $\&$ Lu WQ. A novel germline mutation in SDHA identified in a rare case of gastrointestinal stromal tumor complicated with renal cell carcinoma. International Journal of Clinical and Experimental Pathology 20158 12188-12197.

12 Jha A, de Luna K, Balili CA, Millo C, Paraiso CA, Ling A, Gonzales MK, Viana B, Alrezk R, Adams KT, et al. Clinical, diagnostic, and treatment characteristics of SDHA-related metastatic pheochromocytoma and paraganglioma. Frontiers in Oncology 20199 53. (https://doi.org/10.3389/fonc.2019.00053)

13 Warren AY \& Harrison D. WHO/ISUP classification, grading and pathological staging of renal cell carcinoma: standards and controversies. World Journal of Urology 201836 1913-1926. (https:// doi.org/10.1007/s00345-018-2447-8)

14 Korpershoek E, Favier J, Gaal J, Burnichon N, van Gessel B, Oudijk L, Badoual C, Gadessaud N, Venisse A, Bayley JP, et al. SDHA immunohistochemistry detects germline SDHA gene mutations in apparently sporadic paragangliomas and pheochromocytomas. Journal of Clinical Endocrinology and Metabolism 201196 E1472-E1476. (https://doi.org/10.1210/jc.2011-1043)

15 Killian JK, Miettinen M, Walker RL, Wang Y, Zhu YJ, Waterfall JJ, Noyes N, Retnakumar P, Yang Z, Smith WI, et al. Recurrent epimutation of SDHC in gastrointestinal stromal tumors. Science Translational Medicine 20146 268ra177. (https://doi.org/10.1126/ scitranslmed.3009961)

16 Maniam P, Zhou K, Lonergan M, Berg JN, Goudie DR \& Newey PJ. Pathogenicity and penetrance of germline SDHA variants in pheochromocytoma and paraganglioma (PPGL). Journal of the Endocrine Society 20182 806-816. (https://doi.org/10.1210/js.201800120)

17 Gill AJ. Succinate dehydrogenase (SDH) and mitochondrial driven neoplasia. Pathology 201244 285-292. (https://doi.org/10.1097/ PAT.0b013e3283539932)

18 Ricketts C, Woodward ER, Killick P, Morris MR, Astuti D, Latif F $\&$ Maher ER. Germline SDHB mutations and familial renal cell carcinoma. Journal of the National Cancer Institute 2008100 1260-1262. (https://doi.org/10.1093/jnci/djn254)

19 Williamson SR, Eble JN, Amin MB, Gupta NS, Smith SC, Sholl LM, Montironi R, Hirsch MS \& Hornick JL. Succinate dehydrogenasedeficient renal cell carcinoma: detailed characterization of 11 tumors defining a unique subtype of renal cell carcinoma. Modern Pathology 201528 80-94. (https://doi.org/10.1038/modpathol.2014.86)

Received in final form 23 February 2021

Accepted 2 March 2021 